\title{
OPPORTUNISTIC SENSING: UNATTENDED ACOUSTIC SENSOR SELECTION USING CROWDSOURCING MODELS
}

\author{
Po-Sen Huang ${ }^{\dagger}$, Mark Hasegawa-Johnson ${ }^{\dagger}$, Wotao Yin ${ }^{\ddagger}$, Thomas S. Huang ${ }^{\dagger}$ \\ ${ }^{\dagger}$ Department of Electrical and Computer Engineering, University of Illinois at Urbana-Champaign, USA \\ ${ }^{\ddagger}$ Department of Computational and Applied Mathematics, Rice University, U.S.A. \\ \{huang146, jhasegaw, t-huangl\}@illinois.edu, wotao.yin@rice.edu
}

\begin{abstract}
Unattended wireless sensor networks have been widely used in many applications. This paper proposes automatic sensor selection methods based on crowdsourcing models in the Opportunistic Sensing framework, with applications to unattended acoustic sensor selection. Precisely, we propose two sensor selection criteria and solve them via greedy algorithm and quadratic assignment. Our proposed method achieves, on average, $5.64 \%$ higher accuracy than the traditional approach under sparse reliability conditions.
\end{abstract}

Index Terms - Crowdsourcing models, Quadratic Assignment Problem, Unattended Sensor Networks, Opportunistic Sensing, Cooperative Sensing

\section{INTRODUCTION}

Unattended wireless sensor networks have been widely used in many applications such as target detection, classification, tracking, surveillance, and situation awareness $[1,2]$. Unattended wireless sensor networks have advantages over isolated sensor arrays for extended coverage and improved performance. It is possible to have a large number of sensors deployed randomly to cover remote or hostile regions through air-drop or gun projection so that a certain region can be monitored during days and nights [3].

In addition to sensor networks' performance (e.g. classification accuracy), energy efficiency is a critical concern in unattended wireless sensor networks. Video sensors consume large amounts of power and require a large volume for storage. Hence, it is preferable to use non-imaging sensors, since they tend to use small amounts of power and are long-lasting. Further, for energy conservation, it is desirable to have sensors actively listen (active mode) to the environment only when there are targets present, and to have sensors mostly quiescent (periodic listen and sleep mode) when there is no target present $[4,5]$. Moreover, in noisy conditions, in order to reduce errors, we would like to have a sensor selection framework so that we only activate reliable sensors and make a decision from their responses, while leaving unreliable sensors in the periodic listen and sleep mode.

The idea of selecting reliable sensors can be generalized into the Opportunistic Sensing (OS) framework. The goal of OS is to have an adaptive network that automatically finds objective-driven, scenario-dependent opportunities with optimized performance. Specifically, OS automatically discovers and selects sensing platforms and sensor modalities based on an operational scenario, determines the appropriate set of features and optimal means for data collection based on these features, obtains missing information by querying resources available, and uses appropriate methods to fuse multi-modal data [6].

In this paper, we focus on the automatic selecting of sensing platforms and sensor modalities aspect of OS; that is, given energy constraints, we want to select the minimum number of sensors while maintaining a certain quality of service. Specifically, we propose a general sensor selection framework based on crowdsourcing models with applications to unattended acoustic sensor selection, and we propose two objective functions solved with greedy algorithm and quadratic assignment.

The remainder of this paper is organized as follows: Section 2 will briefly describe related work. Section 3 presents the sensor selection problem formulation, its relation to crowdsourcing models, and the optimization problems. The experiment setups, along with results, are described in Section 4. Section 5 concludes the paper and discusses future work.

\section{PREVIOUS WORK}

The goal of sensor selection in a sensor network is to select sensors that capture informative and reliable data. This paper proposes a sensor selection framework with applications to unattended wireless acoustic sensor networks; that is, our proposed methods select a small number of microphones based on signal probability distributions implied by the network. For an acoustic sensor array/network, the problem of 
optimally fusing signals from multiple microphones is one of the classical problems of signal processing [7], but few papers have considered the problem of selecting a small number of microphones while leaving the remainder turned off. Conversely, many papers consider power management strategies for distributed sensor networks [8], but few consider the particular spatiotemporal signal probability distributions implied by a network of loosely synchronized microphones.

The ideas of selecting informative data and selecting acoustic sensors are also described in [9, 10] and in [3], respectively. Huang et al. [9, 10] proposed a framework of actively sensing and fusing information from acoustic, ultrasonic, and seismic sensors for personnel/animal detection. The authors proposed an active sensing algorithm to actively select highly informative data based on the energy level of seismic sensors. Instead of selecting informative data from a multimodal sensor, this paper further considers the acoustic sensor network case and focuses on selecting reliable sensors to make reliable decisions. Zhang et al. presented an acoustic sensor node selection algorithm to select a set of sensor nodes for source localization and target tracking [3]. Our approach, on the other hand, considers optimizing objective functions using crowdsourcing models for sensor selection in a one-shot scenario.

\section{SENSOR SELECTION}

In this section, we describe the sensor selection problem formulation with applications to unattended wireless acoustic sensor networks, its relation to crowdsourcing models, and the optimization problems.

\subsection{Problem Formulation}

The unattended wireless acoustic sensor network is used for detecting a ground target (e.g. a tank or other vehicle) in the region covered by the sensor network. Each sensor node in the network consists of a power supply/battery, a microphone, and an information-exchanging wireless communication interface.

Suppose that there are $N$ sensor nodes deployed in a region and there is a high level of background noise (e.g. wind sounds) with uniform power spectrum everywhere in the region. Assume that there is a single ground target, which makes sounds. For audio signals, the drop of sound intensity follows the inverse distance square law. Given a target in the region covered by the sensor network, each sensor node can estimate its reliability and make a decision about the presence/absence of the target based on its observed signals. The details are described in Sec. 3.3 below. The goal is to have an automatic sensor selection criterion such that a final decision is made based on the aggregated decision from selected reliable sensors, and unreliable sensors are set to periodic listen and sleep mode for energy conservation. Note that, for each
Fig. 1. An example of a crowdsourcing model, where there are $m$ tasks $t_{i}, 1 \leq i \leq m$, and $n$ workers $w_{j}, 1 \leq j \leq n$.

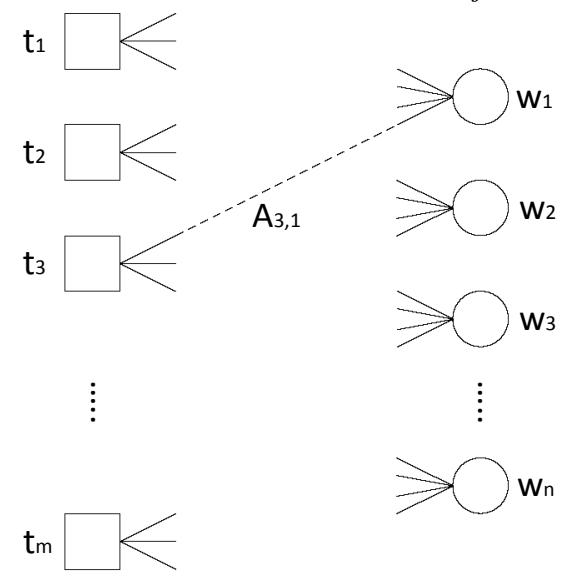

sensor node, the reliability estimation can be done in the listen phase of the periodic listen and sleep mode of the sensors within a short period of time, and that the selected sensors are set to active mode for further monitoring.

\subsection{Crowdsourcing Model}

The problem formulation can be interpreted using crowdsourcing models [11]. We can think of each sensor as a worker and each target as a task. Consider the case that we need to pay a certain fee when we assign a task to a worker, that is, turn on a sensor to detect a target. Given a budget constraint, we would like to have a minimum number of workers assigned to the given task, with reliable prediction accuracy.

Suppose there are a set of $m$ tasks/events/targets $\left\{t_{i}\right\}_{i \in[m]}$ with true answer $s_{i} \in\{1,-1\}$. For example, the label corresponds to the existence of a target $(+1)$ or not $(-1)$. Then, $n$ workers are selected from the crowd for these tasks. Figure 1 shows the idea of assigning $m$ tasks to $n$ workers.

When a task is assigned to a worker, the answer from the worker might be inaccurate. We let $A_{i j} \in\{ \pm 1\}$ denote the answer for task $t_{i}$ assigned to worker $w_{j}$. Since some workers are more reliable than others, we can model the workers' reliability by $p_{i j} \in[0,1]$. To be specific, the answer of worker $w_{j}$ to task $t_{i}$ is

$$
A_{i j}= \begin{cases}s_{i}, & \text { with probability } p_{i j} \\ -s_{i}, & \text { with probability } 1-p_{i j} \\ 0, & t_{i} \text { is not assigned to } w_{j}\end{cases}
$$

For an acoustic signal, the sound power drops $6 \mathrm{~dB}$ every time the distance doubles. Therefore, the error probability of a worker depends on the distance between the task target and the sensor. Note that in reality, e.g. in an open field, there is a high level of background noise such as wind 
Fig. 2. Sensor reliability as a function of sensor location. An example scenario of a target located at coordinates $(7.5,4.5)$.

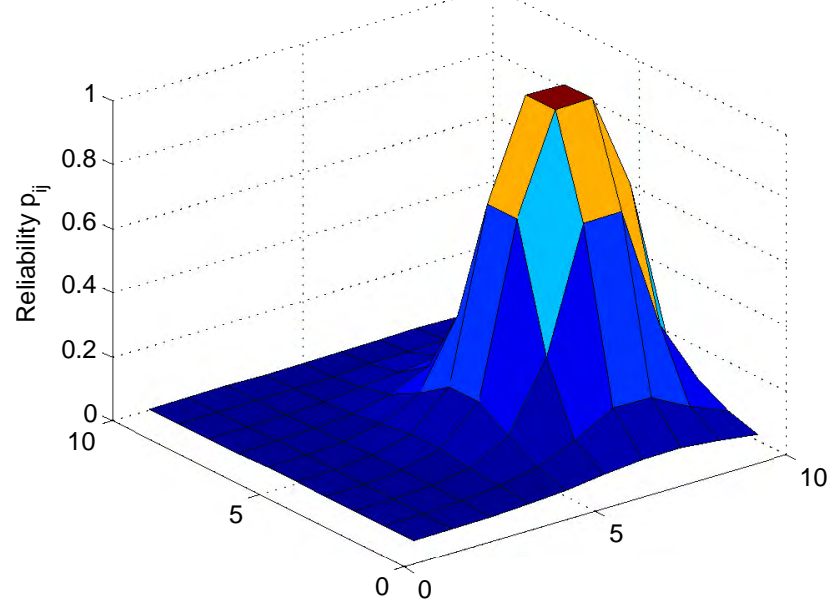

sounds. Figure 2 illustrates a target making a sound at location $(7.5,4.5)$ and sensor reliability dropping in distance. Therefore, selecting sensors based on the sound energy is not reliable. Precisely, in the acoustic sensor network scenario, the reliability of each sensor/worker is modeled by the error probability of a signal classifier distinguishing two hypotheses, viz. $H_{0}: s[n]=v[n]$ vs. $H_{1}: s[n]=d[n]+v[n]$, where $d[n]$ is for simplicity assumed to be a known signal of known duration, and $v[n]$ is a Gaussian disturbance. By thresholding the output of a matched filter, assuming equally likely hypotheses, one can achieve the Bayes minimum error rate $1-p_{i j}=C \exp (-\mathrm{SNR})=a \exp \left(-\frac{1}{r^{2}}\right)$, where $r$ is distance from sensor $j$ to target $i$, and $C$ and $a$ are constants. In this setting, only a few sensors that are close to the target can have reliability $p_{i j}>0.5$. For each sensor, the actual distance to the target is unknown (i.e., ground truth $p_{i j}$ is unknown), but each sensor can estimate the distance according to the estimated signal-to-noise ratio, $\widehat{\mathrm{SNR}}$. From the estimated distance information, sensor $w_{j}$ has an estimated reliability $\hat{p}_{i j}$ for task $t_{i}$. Each sensor models $p_{i j}$ as a random variable $\gamma_{i j}$. For task $t_{i}$, the reliability of workers $\left\{\gamma_{i j}\right\}_{1 \leq j \leq n}$ is a set of random variables taking values in $[0,1]$. Previous applications of the crowdsourcing model assume high average reliability. For example, [11] assumes that $\mathbb{E}\left[2 \gamma_{i j}-1\right]>0$. We deal with the situation where most sensors have low reliability, namely, $\left(\mathbb{E}\left[2 \gamma_{i j}-1\right]<0\right)$. In this setting, the reliable sensors for each given target are sparse.

\subsection{Sensor Selection Objectives}

Given the crowdsourcing model under the condition of sparse reliable sensors, we propose our sensor selection model as follows. We assume that the target location is uniformly distributed. We define the signal as $d[n]$, noise as $v[n]$, and ob- served signal as $s[n]=d[n]+v[n]$. The ground true reliability $p_{i j}$ and the estimated reliability $\hat{p}_{i j}$ follow $p_{i j}=$ $1-C \exp (-\mathrm{SNR})$ and $\hat{p}_{i j}=1-C \exp (-\widehat{\mathrm{SNR}})$, respectively, where SNR $=\frac{N^{-1} \sum_{n} d[n]^{2}}{\sigma_{v}^{2}}$ and $\widehat{\mathrm{SNR}}=\frac{N^{-1} \sum_{n} s[n]^{2}}{\hat{\sigma}_{v}^{2}}-1$, $N$ is the observation window length, and $\sigma_{v}$ and $\hat{\sigma}_{v}$ are the standard deviation of noise $v[n]$ and estimated noise $\hat{v}[n]$, respectively. The constant $C$ is set to 1 in this paper.

We propose two objective functions to select $J$ sensors out of $n$ total sensors (the set of selected sensors is denoted as $\mathcal{J}$, where $|\mathcal{J}|=J$ ) for each task $t_{i}$ :

- Select $J$ sensors such that the probability of success is maximized

$$
J=\underset{J, \mathcal{J}:|\mathcal{J}|=J}{\operatorname{argmax}} P_{\text {success }_{j \in \mathcal{J}}}\left(\hat{p}_{i j}\right)
$$

where $P_{\text {success }}$ is the success probability of a majority voting scheme, for example, given $p_{1}, p_{2}$, and $p_{3}$, the probability of success is modeled as $P_{\text {success }_{j \in\{1,2,3\}}}\left(p_{j}\right)=$ $p_{1} p_{2} p_{3}+p_{1} p_{2}\left(1-p_{3}\right)+p_{2} p_{3}\left(1-p_{1}\right)+p_{1} p_{3}\left(1-p_{2}\right)$.

- Select $J$ sensors such that the probability of average reliability less than a threshold $\alpha$ is minimized

$$
J=\underset{J, \mathcal{J}:|\mathcal{J}|=J}{\operatorname{argmin}} P\left(\frac{1}{J} \sum_{j \in \mathcal{J}} \gamma_{i j}<\alpha \mid \hat{\gamma}_{i\{:\}}\right)
$$

where, by Bayes' rule, $P\left(\gamma_{\boldsymbol{i j}} \mid \hat{\gamma}_{\boldsymbol{i j}}\right) \propto P\left(\gamma_{\boldsymbol{i j}}\right) \times$ $P\left(\hat{\gamma}_{i j} \mid \gamma_{i j}\right)$, and $\hat{\gamma}_{i j}$ is an external confidence estimator that can look at each measurement. Given that $p_{i j}=1-C \exp (-\mathrm{SNR})=1-a \exp \left(-\frac{1}{r^{2}}\right)$, and assuming uniformly distributed target location, so that $P(r \leq R) \propto R^{2}$, it can be shown that the PDF of random variable $\gamma_{i j}$ has a roughly power law formula. In this article, we assume a simplified power law, we let $\gamma_{i j}$ follow the distribution $P\left(\gamma_{i j}=p_{i j}\right) \propto \frac{1}{p_{i j}}$. Once $\gamma_{i j}$ is generated, $\gamma_{i j}$ serves as the parameter of the binomial distribution generating the number of successes worker $w_{j}$ achieves in performing task $t_{i}$, namely, random variable $\boldsymbol{X}_{i j} \sim B\left(N, \boldsymbol{\gamma}_{i j}\right)$. The maximum likelihood estimate of $\gamma_{i j}$ is $\hat{\gamma}_{i j}=\boldsymbol{X}_{i j} / N$. This estimate is itself a random variable. Precisely, $P\left(\hat{\gamma}_{i j} \mid \gamma_{i j}\right)$ follows a scaled binomial distribution. We are interested in $P\left(\gamma_{i j} \mid \hat{\gamma}_{i j}=\hat{p}_{i j}\right)$ following the Bayes rule. The parameter $N$ is set to 2 in this paper. Figure 3 illustrates the two generative processes as a Bayesian net.

\subsection{Optimization}

\subsubsection{Greedy Approach}

Equations (2) and (3) can be solved using a greedy algorithm. Instead of combinatorially choosing $J$ sensors out of $n$ total sensors, we can first sort $\hat{p}_{i\{:\}}$ from high to low, and then 
Fig. 3. An illustration of the two generative processes as a Bayesian net. $\gamma_{i j}, \boldsymbol{X}_{i j}$, and $\hat{\gamma}_{i j}$ are random variables. The shaded nodes are observed.

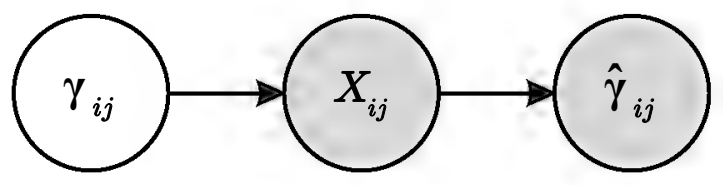

select top $J$ sensors to form set $\mathcal{J}$ according to the objection functions. Specifically, in Eq. (2), we select $J$ sensors to maximize the probability of success according to the estimate probabilities $\hat{p}_{i\{:\}}$. In Eq. (3), we define $S_{p}=\sum_{j \in \mathcal{J}} \boldsymbol{\gamma}_{\boldsymbol{i j}}$, $\hat{S}_{p}=\hat{\gamma}_{i\{:\}}$, and $M_{S_{p}}(t)$ is the moment generating function of $S_{p}$, given $\hat{S}_{p}$. The one-sided Chebyshev bound specifies that

$$
P(X-\mu \geq k \sigma) \leq \frac{1}{1+k^{2}}
$$

Thus we can find

$$
\begin{aligned}
J & =\underset{J, \mathcal{J}:|\mathcal{J}|=J}{\operatorname{argmin}} P\left(S_{p}<J \alpha \mid \hat{S}_{p}\right) \\
& \approx \underset{J, \mathcal{J}:|\mathcal{J}|=J}{\operatorname{argmin}}\left\{\frac{\left(J \alpha-E\left[S_{p} \mid \hat{S}_{p}\right]\right)^{2}}{\operatorname{Var}\left(S_{p} \mid \hat{S}_{p}\right)+\left(J \alpha-E\left[S_{p} \mid \hat{S}_{p}\right]\right)^{2}}\right\}
\end{aligned}
$$

where the calculations are done using the property that the moment generating function for $S_{p}$ is the multiplication of the moment generating functions of each of the $\gamma_{i j}$, given $\hat{\gamma}_{i j}$. The mean and variance are $E\left[S_{p} \mid \hat{S}_{p}\right]=\left.\frac{d M_{S_{p}}(t)}{d t}\right|_{t=0}$, $\operatorname{Var}\left(S_{p} \mid \hat{S}_{p}\right)=\left.\frac{d^{2} M_{S_{p}}(t)}{d t^{2}}\right|_{t=0}-E\left[S_{p} \mid \hat{S}_{p}\right]^{2}$, respectively.

\subsubsection{Quadratic Assignment Problem}

Equation (3) can be solved globally by formulating our objective function as a quadratic assignment problem (QAP) $[12,13]$. Given matrices $A, B, C \in \mathbb{R}^{\tilde{n} \times \tilde{n}}$, the QAP minimizes a quadratic function over a permutation matrix $X$ as shown in Eq. (5).

$$
\begin{gathered}
\min _{X \in \mathbb{R}_{\tilde{n} \times \tilde{n}}} \operatorname{tr}\left(A X B^{T} X^{T}\right)+\operatorname{tr}\left(C^{T} X\right) \\
\text { subject to } X^{T} X=I \\
X \geq 0
\end{gathered}
$$

where the permutation matrix $X=\left[x_{j i}\right] \in\{0,1\}^{\tilde{n} \times \tilde{n}}$ is an assignment matrix, where each row and each column have only one nonzero entry of 1 , and $x_{j i}=1$ represents assigning worker $w_{j}$ to task $t_{i}$. Note that $X$ is a permutation matrix of size $\tilde{n}$ by $\tilde{n}$ if and only if the following conditions hold: $X \in$ $\mathbb{R}^{\tilde{n} \times \tilde{n}}, X^{T} X=I$, and $X \geq 0$ (component-wise positive).
The QAP objective is from

$$
\begin{array}{r}
\sum_{1 \leq i, j, k, l \leq \tilde{n}} a_{i, j} b_{k, l} x_{i, k} x_{j, l}+\sum_{1 \leq i, j \leq \tilde{n}} c_{i, j} x_{i, j} \\
=\operatorname{tr}\left(A X B^{T} X^{T}\right)+\operatorname{tr}\left(C^{T} X\right)
\end{array}
$$

where $a_{i, j}$ is the cost between workers $w_{i}$ and $w_{j}, b_{k, l}$ is the costs between tasks $t_{k}$ and $t_{l}$, and $c_{i, j}$ is the cost between worker $w_{i}$ and task $t_{j}$.

Hence, Eq. (4) can be written as selecting $X$, such that:

$$
\begin{gathered}
X=\underset{X}{\operatorname{argmin}}\left\{\frac{\left(J \alpha-\mathbb{E}\left[S_{p} \mid \hat{S}_{p}\right]\right)^{2}}{\operatorname{Var}\left(S_{p} \mid \hat{S}_{p}\right)+\left(J \alpha-\mathbb{E}\left[S_{p} \mid \hat{S}_{p}\right]\right)^{2}}\right\} \\
\approx \underset{X}{\operatorname{argmin}}\left[\mathbb{E}\left[S_{p} \mid \hat{S}_{p}\right]^{2}-2 J \alpha \mathbb{E}\left[S_{p} \mid \hat{S}_{p}\right]\right. \\
\left.+J^{2} \alpha^{2}-\lambda \operatorname{Var}\left(S_{p} \mid \hat{S}_{p}\right)\right]
\end{gathered}
$$

where the latter equation is relaxed using a Lagrangian multiplier $\lambda$. The number of active sensors $J^{1}$, the mean $\mathbb{E}\left[S_{p} \mid \hat{S}_{p}\right]$, and variance $\operatorname{Var}\left(S_{p} \mid \hat{S}_{p}\right)$, by adding dummy tasks and workers, can be formulated as the following:

$$
\begin{aligned}
\mathbb{E}\left[S_{p} \mid \hat{S}_{p}\right]= & {\left[\begin{array}{c}
\mathbb{E}\left[\gamma_{\boldsymbol{i} \mathbf{1}} \mid \hat{\gamma}_{\boldsymbol{i 1}}\right] \\
\vdots \\
\mathbb{E}\left[\gamma_{\boldsymbol{i n}} \mid \hat{\gamma}_{\boldsymbol{i n}}\right] \\
0 \\
\vdots \\
0
\end{array}\right]^{T} X\left[\begin{array}{c}
1 \\
\vdots \\
1 \\
0 \\
\vdots \\
0
\end{array}\right] } \\
\operatorname{Var}\left(S_{p} \mid \hat{S}_{p}\right)= & {\left[\begin{array}{c}
\operatorname{Var}\left(\gamma_{\boldsymbol{i n}} \mid \hat{\gamma}_{\boldsymbol{i n}}\right) \\
\vdots \\
\operatorname{Var}\left(\gamma_{i n} \mid \hat{\gamma}_{i n}\right) \\
0 \\
\vdots \\
0
\end{array}\right]^{T} X\left[\begin{array}{c}
1 \\
\vdots \\
1 \\
0 \\
\vdots \\
0
\end{array}\right] }
\end{aligned}
$$

$$
J=\left[\begin{array}{c}
1 \\
\vdots \\
1 \\
0 \\
\vdots \\
0
\end{array}\right]^{T} X\left[\begin{array}{c}
1 \\
\vdots \\
1 \\
0 \\
\vdots \\
0
\end{array}\right]
$$

where $X \in \mathbb{R}^{\tilde{n} \times \tilde{n}}, \tilde{n}=2 n, n$ is the total number of sensors. By matrix trace tricks:

\footnotetext{
${ }^{1}$ Note that, in the QAP approach, the set $\mathcal{J}$ is selected automatically.
} 


$$
\begin{aligned}
\left(a^{T} X b\right)\left(c^{T} X d\right) & =\left(a^{T} X b\right)^{T}\left(c^{T} X d\right) \\
& =\operatorname{tr}\left(b^{T} X a c^{T} X d\right) \\
& =\operatorname{tr}\left(a c^{T} X d b^{T} X^{T}\right)
\end{aligned}
$$

where $a, b \in \mathbb{R}^{\tilde{n}}$ are vectors, we can formulate Eq. (6) into QAP formulation in Eq. (5).

\section{EXPERIMENTS}

In our experiment, simulations are used to evaluate the effectiveness of our proposed sensor selection criteria. In our simulation, there are a hundred sensors and each one is at the grid point of a $10 \times 10$ Cartesian grid. $N=1000$ targets/tasks occur independently at random positions in the grid. For each sensor, the signal at the $j$-th sensor $d_{j}[n]=d_{\text {target }}[n] / r_{j}^{2}$, where $d_{\text {target }}[n]$ is the original signal generated from the target and $r_{j}$ is the distance from the target to the $j$-th sensor. In each setup, we generate white Gaussian noise uniformly for each sensor at SNR = $\{-6.51,-5.21,-4.34,-3.91,-3.47,-3.04,-2.17,-1.30$, $0.00\} \mathrm{dB}$, where the corresponding highest reliabilities at the closest sensor are $p_{i j}=\{0.59,0.70,0.77,0.80,0.83,0.86$, $0.91,0.94,0.98\}$, respectively. In each case, the final decision of each task is determined by majority voting using the answers from the selected sensors.

We compare the accuracy in the following cases at different SNRs:

- Select the highest $\hat{p}_{i\{:\}}$ sensor, which is the baseline approach. (Denoted as the Top case.)

- Select $J$ sensors to maximize probability of success using greedy approach. (Denoted as the SuccessProb case.)

- Select $J$ sensors to minimize probability that average reliability is less than a threshold using greedy approach. (Denoted as the AvgProb-Greedy-threshold case.)

- Select $J$ sensors to minimize probability that average reliability is less than a threshold using QAP. (Denoted as the AvgProb-QAP-threshold case.)

The experimental results are shown in Fig. 4. There are some interesting observations. First, selecting the highest estimated reliability sensor is not the best solution compared with our proposed methods. Second, maximizing probability of success achieves the highest accuracy in many cases, $\mathrm{SNR}=\{-6.51,-5.21,-2.17,-1.30,0.00\} \mathrm{dB}$. Third, minimizing probability that average reliability is than a threshold also achieves competitive results, especially in the QAP formulation. Moreover, QAP formulation achieves higher accuracy compared with the greedy approach, since it provides a way to solve the assignment problem globally.
Fig. 4. Experimental results of different sensor selection criteria. The Top case is the baseline.

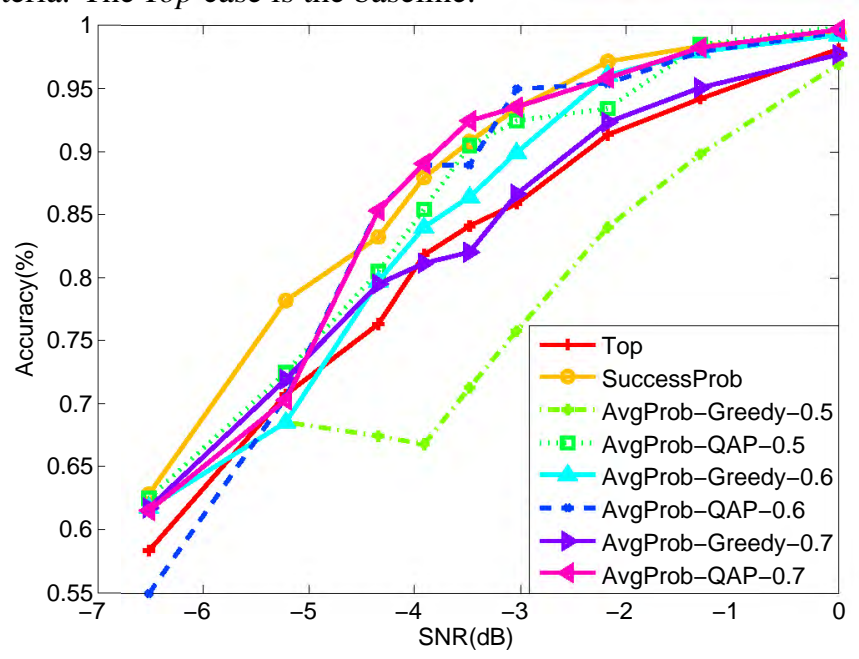

Furthermore, we use a paired-sample t-test to further examine the significance of our results [14]. We pair the results of the SuccessProb and the Top case. The p-values at different SNRs are shown in Table 1. Note that the highest p-value is $13.9 \%$ at SNR $=-5.21 \mathrm{~dB}$, and other values are less than $5 \%$, which suggests that the SuccessProb algorithm significantly outperforms the baseline at all noise levels ( $5.64 \%$ on average) except SNR $=-5.21 \mathrm{~dB}$.

\section{CONCLUSION}

In this paper, we study opportunistic sensing for automatic selection of acoustic sensors. We model the sensor selection problem using crowdsourcing models under sparse reliability constraints. We propose two optimization objective functions with greedy approach and quadratic assignment.

There are many possibilities for extending this work. For example, in addition to the current one-shot sensor selection problem, we can further consider the sensor selection in a time-varying scenario. Also, in addition to acoustic sensors, our proposed models and algorithms can be generalized to other unattended sensor networks.

\section{ACKNOWLEDGEMENTS}

This research was supported by U.S. ARL and ARO under grant number W911NF-09-1-0383. We thank Sewoong Oh for helpful discussions in crowdsourcing models.

\section{REFERENCES}

[1] T. Damarla, "Sensor fusion for ISR assets," 2010, SPIE. 
Table 1. p-values (\%) of a paired-sample t-test between the SuccessProb and the Top case. The SuccessProb case significantly outperforms the Top case (at $p<0.05$ ) in all cases except SNR $=-5.21 \mathrm{~dB}$.

\begin{tabular}{|c||c|c|c|c|c|c|c|c|c|}
\hline SNR (dB) & -6.51 & -5.21 & -4.34 & -3.91 & -3.47 & -3.04 & -2.17 & -1.30 & 0.00 \\
\hline $\mathrm{p}$ & $4.19 \times 10^{-1}$ & $1.39 \times 10^{1}$ & $3.81 \times 10^{-5}$ & $8.99 \times 10^{-7}$ & $1.13 \times 10^{-2}$ & $3.44 \times 10^{-5}$ & $3.78 \times 10^{-5}$ & $1.41 \times 10^{-4}$ & $6.52 \times 10^{-2}$ \\
\hline
\end{tabular}

[2] T. Damarla, L. Kaplan, and A. Chan, "Human infrastructure \& human activity detection," in International Conference on Information Fusion, 2007, pp. 1-8.

[3] J. Zhang, M. Walpola, D. Roelant, H. Zhu, and K. Yen, "Self-organization of unattended wireless acoustic sensor networks for ground target tracking," Pervasive and Mobile Computing, vol. 5, no. 2, pp. 148-164, 2009.

[4] F. Bennett, D. Clarke, J.B. Evans, A. Hopper, A. Jones, and D. Leask, "Piconet: Embedded mobile networking," IEEE Personal Communications, vol. 4, no. 5, pp. 8-15, 1997.

[5] W. Ye, J. Heidemann, and D. Estrin, "An energyefficient MAC protocol for wireless sensor networks," in Proceedings of INFOCOM, 2002, vol. 3, pp. 15671576.

[6] A. Kapadia, D. Kotz, and N. Triandopoulos, "Opportunistic sensing: Security challenges for the new paradigm," in Communication Systems and Networks and Workshops, Jan. 2009, pp. 1-10.

[7] P. Aarabi, "The fusion of distributed microphone arrays for sound localization," EURASIP J. Appl. Signal Process., pp. 338-347, Jan. 2003.

[8] D. W. Carman, P. S. Kruus, and B. J. Matt, "Constraints and approaches for distributed sensor network security," Tech. Rep., NAI Labs, The Security Research Division Network Associates, Inc., Sep. 2000.

[9] P.-S. Huang, M. Hasegawa-Johnson, and T. Damarla, "Exemplar selection methods to distinguish human from animal footsteps," in Human and Light Vehicle Detection Workshop, 2011.

[10] P.-S. Huang, T. Damarla, and M. Hasegawa-Johnson, "Multi-sensory features for personnel detection at border crossings," in International Conference on Information Fusion, July 2011, pp. 1-8.

[11] D. R. Karger, S. Oh, and D. Shah, "Iterative learning for reliable crowdsourcing systems," in Advances in Neural Information Processing Systems (NIPS), 2011, pp. 1953-1961.

[12] R. E. Burkard, S. E. Karisch, and F. Rendl, “QAPLIB - a quadratic assignment problemlibrary," J. of Global Optimization, vol. 10, no. 4, pp. 391-403, 1997.
[13] Z. Wen and W. Yin, "A feasible method for optimization with orthogonality constraints," Tech. Rep., Rice University, 2010.

[14] L. Gillick and S. Cox, "Some statistical issues in the comparison of speech recognition algorithms," in Proc. of ICASSP, 1989, pp. 532-535. 